\title{
Idiopathic Pulmonary Fibrosis With Emphysema: Evidence of Synergy Among Emphysema and Idiopathic Pulmonary Fibrosis in Smokers
}

\author{
Patrick D Mitchell MRCPI, Jeeban P Das MRCPI, David J Murphy FFR RCSI, \\ Michael P Keane PhD FRCPI, Seamas C Donnelly MD FRCPI, \\ Jonathan D Dodd FFR RCSI, and Marcus W Butler MD FRCPI
}

BACKGROUND: Emphysema and fibrosis, typically the idiopathic pulmonary fibrosis (IPF) form of usual interstitial pneumonia (UIP), can co-exist as combined pulmonary fibrosis emphysema (CPFE). It is unknown whether there is a pathobiologic basis for CPFE beyond the coexistence of fibrosis and emphysema. The aim of this study was to ascertain radiologic differences in severity of fibrosis and emphysema in smokers with IPF versus other forms of UIP. METHODS: Computed tomography thorax images were prospectively rescored in retrospectively identified smokers (minimum 5-pack-year history) with radiologic UIP (any etiology). Radiologic severity (emphyse$\mathrm{ma} /$ fibrosis/reticulation) was scored in consensus by two radiologists, blinded to clinical details, across 5 lung regional levels, and then correlated with clinical data. RESULTS: For the whole cohort (IPF, $n=102$; non-IPF UIP [mainly rheumatoid arthritis/asbestosis/scleroderma], $n=30$ ), IPF and non-IPF UIP smokers were similar regarding pack-year, age, gender, and lung function $(P>$.1). IPF smokers had greater whole lung fibrosis and reticulation scores $(P<.04$ in all cases). CPFE was present in $n=61$ (IPF, $n=49$; non-IPF UIP, $n=12$ ). Compared with smokers with non-IPF CPFE, smokers with IPF and emphysema (IPFE) were similar regarding confounders $(P>.1)$. There were significantly greater regional reticulation severity $(P=.009)$, cumulative emphysema severity $(P=.04)$, and cumulative reticulation severity $(P<.001)$ scores in IPFE versus non-IPF CPFE. CONCLUSIONS: When controlled for confounders, smokers with IPFE have worse radiologic CPFE than other smokers with non-IPF UIP and emphysema, suggesting an interactive synergy among IPF, emphysema, and smoking, with more extensive emphysema due to either inherent susceptibility and/or traction effects. IPFE should be considered separately from other CPFE in future work. It is currently unknown whether CPFE is a distinct pathobiologic entity; therefore, we identified subjects with radiologic UIP (any etiology) who had been similarly exposed to smoke, and asked whether there are differences in the extent/severity of radiologic fibrosis and/or emphysema in those with IPF versus individuals with non-IPF UIP. Although relevant confounders were similar, IPF smokers had greater whole lung fibrosis and reticulation scores than smokers with secondary forms of UIP, and in the CPFE subgroup, smokers with IPF/emphysema had worse radiologic CPFE findings than smokers with non-IPF UIP/emphysema. It is shown for the first time that relevant confounding variables do not explain the observed excess radiologic severity of emphysema and fibrosis in smokers with IPF compared with smokers with non-IPF UIP, lending support to the hypothesis that there is a pathobiologic mechanism or synergy involved in IPF with emphysema that is distinct from the mere co-existence of UIP and emphysematous processes. Key words: smoking; emphysema; connective tissue disease; idiopathic pulmonary fibrosis; usual interstitial pneumonia; reticulation score. [Respir Care 2015;60(2):259-268. (c) 2015 Daedalus Enterprises] 


\section{Introduction}

The pathological changes of emphysema and pulmonary fibrosis are commonly found in smokers. ${ }^{1}$ Patients who have both these pathologies may have different pulmonary physiology and different outcomes clinically than those with sole pulmonary emphysema or pulmonary fibrosis. ${ }^{2}$ The resulting clinical profile is generally characterized by relatively normal spirometry and lung volumes in the setting of severely impaired gas exchange. ${ }^{3}$ Combined pulmonary fibrosis and emphysema syndrome (CPFE syndrome) is a recognized clinically severe pulmonary disease incorporating both pulmonary emphysema and fibrosis. ${ }^{4,5}$ CPFE syndrome is most frequently found in older males who have a heavy pack-year burden of smoking. ${ }^{6,7}$ The proportion of patients with pulmonary fibrosis who also have emphysema ranges from $8 \%$ to $50.9 \% .^{7-9}$ However, other patterns of fibrotic lung disease have also been reported in conjunction with emphysema. ${ }^{5,10,11} \mathrm{CPFE}$ syndrome has been described in patients with connective tissue disease. ${ }^{12}$ The histological types of pulmonary fibrosis found in CPFE syndrome are typically described as usual interstitial pneumonia (UIP) or nonspecific interstitial pneumonia, with the latter evident in only a minority of reported cases. Primary UIP is synonymous with idiopathic pulmonary fibrosis (IPF) and secondary UIP can occur in the setting of conditions including asbestosis, rheumatoid arthritis, Sjögren's syndrome, dermatomyositis, and polymyositis. ${ }^{13,14}$ There are conflicting reports in the literature of the extent to which IPF and other causes of UIP can be associated with CPFE, some of which are confounded by gender and smoking parameters. ${ }^{15-17}$ Our group postulated that, in smokers with radiologic UIP who have been similarly exposed to smoke, the extent/severity of fibrosis and/or emphysema would be greater in IPF than in individuals with non-IPF UIP.

Drs Mitchell, Das, Murphy, Keane, Donnelly, Dodd, and Butler are affiliated with St Vincent's University Hospital, Dublin; Drs Keane, Donnelly, and Butler are also affiliated with University College Dublin, Dublin, Republic of Ireland.

Drs Mitchell, Das, and Murphy are co-first authors of this paper.

Dr Mitchell presented a version of this paper at the 2013 American Thoracic Society International Conference, held May 17-22, 2013, Philadelphia, Pennsylvania.

The authors have disclosed no conflicts of interest.

Correspondence: Patrick D Mitchell MRCPI, Education and Research Centre, St Vincent's University Hospital, Dublin 4, Republic of Ireland. E-mail: patrickdavidmitchell@gmail.com.

DOI: $10.4187 /$ respcare. 03389

\section{QUICK LOOK}

\section{Current knowledge}

Combined pulmonary fibrosis and emphysema syndrome (CPFE syndrome) is a recognized severe pulmonary disease incorporating both pulmonary emphysema and fibrosis. CPFE syndrome is most frequently seen in older men who have a heavy pack-year burden of smoking. The proportion of patients with pulmonary fibrosis who also have emphysema ranges from $8 \%$ to $50 \%$.

\section{What this paper contributes to our knowledge}

Although relevant confounders were similar, interstitial pulmonary fibrosis smokers had greater whole lung fibrosis and reticulation scores than smokers with secondary forms of usual interstitial pneumonia, and, in the CPFE subgroup, smokers with idiopathic pulmonary fibrosis (IPF)/emphysema had worse radiologic CPFE findings than smokers with non-IPF usual interstitial pneumonia/emphysema.

\section{Methods}

This single-center, retrospective study was conducted at St. Vincent's University Hospital, Dublin, which is a national referral center for interstitial lung disease in Ireland. Following approval from the institutional review board, radiology databases were searched for patients using search terms including UIP, usual interstitial pneumonia, IPF, idiopathic pulmonary fibrosis, honeycombing, asbestosis, and traction bronchiectasis, as well as various collagen vascular lung disease search terms.

Scans earlier than 2006 were disregarded due to lower resolution computed tomography (CT) scanners then in use. Thoracic CT had been acquired on a 64-slice singlesource CT system (Siemens Sensation 64, Siemens Medical Solutions, Forchheim, Germany). CT examinations which were included for analysis included non-contrast high-resolution CT thorax, CT pulmonary angiogram, and contrast-enhanced CT thorax. CTs were acquired from apex to lung base at full inspiration using approximately 120 $\mathrm{KvP}$ and $130 \mathrm{mAs}$. Axial CT slices $(1 \mathrm{~mm})$ were analyzed using lung windows with a width of 1,500 Hounsfield units and center of -500 Hounsfield units. Subjects with a documented and quantified history of current or prior smoking ( $\geq 5$ pack-years) were identified from clinical record review and radiology databases, based on a reported radiologic finding of any form of UIP. Inclusion criteria were a positive (current or former) smoking his- 


\section{IPF WITH EMPHYSEMA}

tory ( $\geq 5$ pack-years) and radiologic diagnosis of probable or definite UIP by established criteria, based on prospective review of thoracic $\mathrm{CT}$ images. ${ }^{18} \mathrm{CT}$ image data sets were loaded into interactive image processing software (Syngo InSpace4D, Siemens Medical Solutions). Two radiologists were blinded to the subjects' clinical details and scored CT thoracic images in consensus using established scoring schemata. Images were reviewed at 5 levels: (1) origin of the great vessels, (2) carina, (3) pulmonary venous confluence, (4) between levels 3 and 5, and (5) $1 \mathrm{~cm}$ above the right hemidiaphragm, and images were scored with respect to percentage of pulmonary parenchymal fibrosis and ground glass opacification, reticulation (coarseness of fibrosis, scored 0-3), and extent of emphysema (scored 0-4). ${ }^{19,20}$ The IPF UIP group had a multidisciplinary team diagnosis of IPF (with no clinical or serological evidence of autoimmune disease, connective tissue disease, or relevant drug/antigen exposure history, including environmental exposure to asbestos). The non-IPF UIP group was composed of subjects with radiologic UIP and a multidisciplinary team diagnosis of rheumatoid arthritis, Sjögren's syndrome, dermatomyositis, polymyositis, or asbestosis according to recognized guidelines. ${ }^{21,22}$ Of the 132 subjects included in the present study, a total of 18 subjects (13.6\%) underwent surgical lung biopsy, and revealed a pathologic finding of UIP (8 subjects in the IPF group and 10 subjects in the non-IPF group). The extent of interstitial lung disease (reticular pattern and ground-glass opacification) was evaluated using a derivation of the pulmonary scoring system described by Desai et al. ${ }^{23}$ The definitions of a reticular pattern and ground-glass opacification were derived from the Fleischner society glossary of terms. ${ }^{24}$ The coarseness of fibrosis was quantified semiquantitatively as follows: 0 , no fibrosis; 1 , fine intralobular fibrosis; 2, microcystic $(<4 \mathrm{~mm})$ reticular pattern; and 3, macrocystic $(>4 \mathrm{~mm})$ reticular pattern. Adjustment was made for cumulative coarseness scores where one or more lung region was unaffected, to prevent spurious underestimation of coarseness due to localized distribution of fibrosis, as previously described, by multiplying cumulative scores by a correction factor of $5 / n$, where $n$ is the number of involved levels. ${ }^{15}$ Overall extent of interstitial lung disease (reticular pattern and ground-glass opacification) was estimated to the nearest 5\%. Each level was assessed in turn for emphysematous changes; these included areas of low pulmonary parenchymal attenuation, areas of lung destruction, and areas of vascular destruction. The extent of emphysema was estimated using a scoring system $(0=<5 \%$ emphysema; $1=5-25 \% ; 2=25-50 \% ; 3=50-$ $75 \% ; 4=75-100 \%$ ) derived from Bankier and colleagues. ${ }^{24-26}$ Correlation of scores with clinical data were performed (after image analysis) by another investigator (MB).
Table 1. Demographic and Lung Physiologic Variables of the Study Population

\begin{tabular}{|c|c|c|c|c|}
\hline Parameter & IPF & Non-IPF & Total UIP & $P^{*}$ \\
\hline Male/female & $70 / 32$ & $19 / 11$ & $89 / 43$ & .66 \\
\hline Age (y) & $73.3 \pm 1.0$ & $69.8 \pm 2.0$ & $72 \pm 0.9$ & .13 \\
\hline Pack-years & $29.3 \pm 1.7$ & $35.1 \pm 3.5$ & $30.6 \pm 1.5$ & .15 \\
\hline \multicolumn{5}{|l|}{ Diagnoses, $n$} \\
\hline IPF & 102 & NA & & \\
\hline RA-ILD & NA & 14 & & \\
\hline Asbestosis & NA & 9 & & \\
\hline Scleroderma & NA & 4 & & \\
\hline Other $\dagger$ & NA & 3 & & \\
\hline \multicolumn{5}{|l|}{ Pulmonary function tests } \\
\hline FVC \% & $81.8 \pm 2.1$ & $78.2 \pm 4.5$ & $80.9 \pm 1.9$ & .54 \\
\hline $\mathrm{FEV}_{1} \%$ & $79.8 \pm 1.9$ & $76.3 \pm 4.7$ & $78.9 \pm 1.9$ & .54 \\
\hline $\mathrm{FEV}_{1} / \mathrm{FVC} \%$ observed & $76.6 \pm 1.3$ & $73.3 \pm 3.1$ & $75.9 \pm 1.2$ & .44 \\
\hline TLC \% predicted & $79.2 \pm 2.3$ & $82.2 \pm 2.7$ & $79.8 \pm 1.8$ & .73 \\
\hline $\mathrm{D}_{\text {LCO }} \%$ predicted & $52.5 \pm 1.6$ & $59.1 \pm 4.6$ & $54.0 \pm 1.6$ & .35 \\
\hline $\begin{array}{l}\text { Mean values } \pm \text { standard errors are } \\
* P \text { values represent Fisher exact te } \\
\dagger \text { Other UIP diagnoses included in } \\
\text { Sjogren's syndrome, and polymyosi } \\
\text { IPF }=\text { idiopathic pulmonary fibrosis } \\
\text { UIP = usual interstitial pneumonia } \\
\text { NA }=\text { not applicable } \\
\text { RA-ILD = rheumatoid arthritis-asso } \\
\text { TLC = total lung capacity } \\
\text { D }_{\text {LCO }} \text { = diffusing capacity of the lu }\end{array}$ & $\begin{array}{l}\text { lown where appli } \\
\text { or Student } t \text { test } \\
\text { es study populatic } \\
\text { is. }\end{array}$ & $\begin{array}{l}\text { cable. } \\
\text { as appropriate. } \\
\text { n were mixed co }\end{array}$ & Inective tissue dis & \\
\hline
\end{tabular}

\section{Statistical Analysis}

Continuous variables are presented as mean \pm SD. Categorical variables are presented as frequencies and percentages. Comparisons of parametric data were performed by Student $t$ test, and comparisons of non-parametric radiologic scores were assessed using a two-tailed independent sample Mann-Whitney U test. All other non-parametric comparisons were made using a two-tailed Fisher exact test. All statistical analyses were performed with SPSS 18.0 software (SPSS, Chicago, Illinois). A value of $P<.05$ was considered significant. For analyses involving multiple simultaneous comparisons (of radiologic scores at 5 different lung levels), correction was made for false discovery using Bonferroni correction.

\section{Results}

A total of 169 subjects $($ male $=119$, female $=50)$ were initially selected based on documented smoking history ( $\geq 5$ pack-years) and radiologic reports of UIP. Following prospective CT scan image review, the blinded radiology investigators excluded 37 subjects ( 35 cases: not probable or definite radiologic UIP; 1 case: diffuse pulmonary metastatic disease; 1 case: unlocatable scan images). This left 132 subjects with radiologic UIP for further anal- 

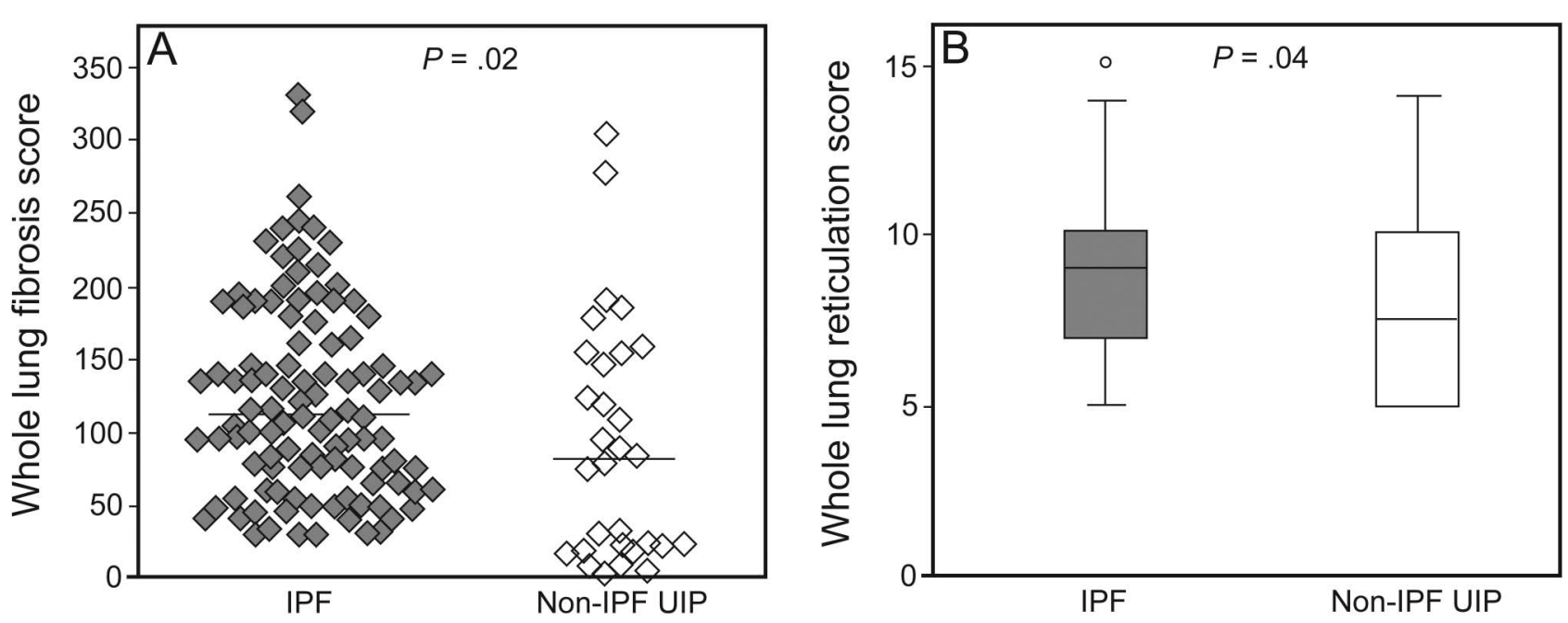

Fig. 1. Radiologic fibrosis scores in IPF and non-IPF UIP groups. A: extent of fibrosis as assessed by cumulative fibrosis scores for all 5 radiologic lung levels per subject. Each diamond represents a subject's cumulative score. Median values are represented by horizontal lines in both groups. B: coarseness of fibrosis shown as cumulative reticulation scores for all 5 radiologic lung levels per subject, with adjustment of raw scores for uninvolved lung levels (see Methods). Box plot represents interquartile range and median, and whiskers represent data within 1.5 interquartile range of lower and upper quartiles. Outliers are shown as open circles. Two-tailed independent sample MannWhitney $U$ test $P$ values are shown. IPF = idiopathic pulmonary fibrosis. UIP $=$ usual interstitial pneumonia.

ysis, $n=102$ with IPF UIP and $n=30$ with non-IPF UIP group (Table 1). The two groups were similar regarding gender, age, and cigarette pack-year burden $(P>.1$ in all cases; Table 1).

First, we asked whether the two similarly smoke-exposed groups differed from one another with respect to extent and coarseness of radiologic fibrosis. The data demonstrated a significantly greater extent (Fig. 1A) and coarseness (Fig. 1B) of fibrosis in IPF UIP compared with nonIPF UIP $(P=.02$ and $P=.03$, respectively). Next, emphysema measurements were assessed. Radiologic emphysema was defined as present if at least one of the 5 radiologic lung levels had $\geq 5 \%$ emphysema. In total, $46 \%$ of the study population met this permissive definition of emphysema, and hence CPFE. Weak nonsignificant trends were observed toward higher prevalence of emphysema in IPF UIP compared with non-IPF UIP subjects $(P=.6$; Fig. 2A $)$ and higher emphysema prevalence in IPF UIP subjects at all 5 lung regions $(P=.5$ at the great vessels, $P=.1$ at the carina, $P=.08$ at the pulmonary vein level, $P=.3$ at point 4 , and $P=.067$ at the right hemidiaphragm; Fig. 2B). As would be expected, the data for lung regional prevalence of emphysema exhibited a progressive decline from lung apex to the diaphragm. Extent of radiologic emphysema was analyzed by summating the emphysema scores obtained at each of the 5 radiologic lung levels. There was a nonsignificant trend toward higher emphysema extent in IPF UIP versus non-IPF UIP $(P=.1$; Fig. 2C). There were concordant trends at all lung levels toward a greater extent of emphysema in IPF compared with non-IPF UIP, attaining unadjusted, conventional sta- tistical significance at the levels of the pulmonary venous confluence $(P=.044)$ and the right hemidiaphragm $(P=.050)$, but statistically insignificant when corrected for multiple testing $(P=.1$, Fig. 2D).

A priori, the CPFE cohort were studied further. A total of 61 subjects met the study's definition of CPFE (two subgroups: IPF UIP/emphysema, $n=49$; and non-IPF UIP/emphysema, $n=12$ ). The two subgroups did not differ in terms of cigarette pack-year, age, or gender $(P=.8$, $P=.1$, and $P=.7$, respectively; Fig. 3A). A nonsignificant trend was seen toward higher fibrosis extent in IPF/emphysema versus non-IPF UIP/emphysema subjects $(P=.1$, Fig. 3B). The fibrosis extent, as expected, became higher toward the lung bases. There were concordant, statistically insignificant trends (Bonferroni corrected) toward higher fibrosis scores in IPF/emphysema subjects than their non-IPF counterparts, strongest nearer the lung apices (Fig. 3C).

Adjusted cumulative reticulation scores (see Methods) revealed coarser fibrosis in IPF/emphysema subjects versus non-IPF UIP/emphysema individuals $(P<.001$; Fig. $4 \mathrm{~A})$. The lung regional analysis of fibrotic coarseness was significantly greater (post-Bonferroni) in the 3 most apical lung regions analyzed for IPF/emphysema subjects, compared with non-IPF counterparts (specifically $P=.009$ at the great vessels, $P<.001$ at the carina, $P<.001$ at the pulmonary vein level, $P=.43$ at point 4 , and $P=.112$ at the right hemidiaphragm; Fig. 4B). Whereas most IPF/emphysema subjects had finer reticulation changes at the level of the great vessels, at all other levels assessed, there were mainly micro- or macrocysts seen. The non-IPF 
A
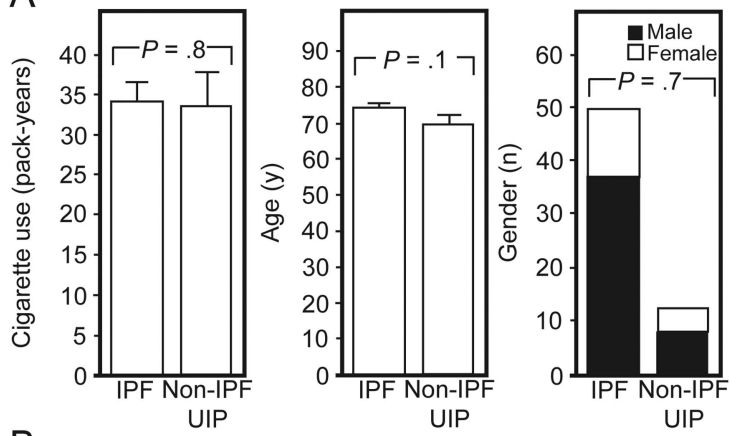$$
\text { 高 }
$$
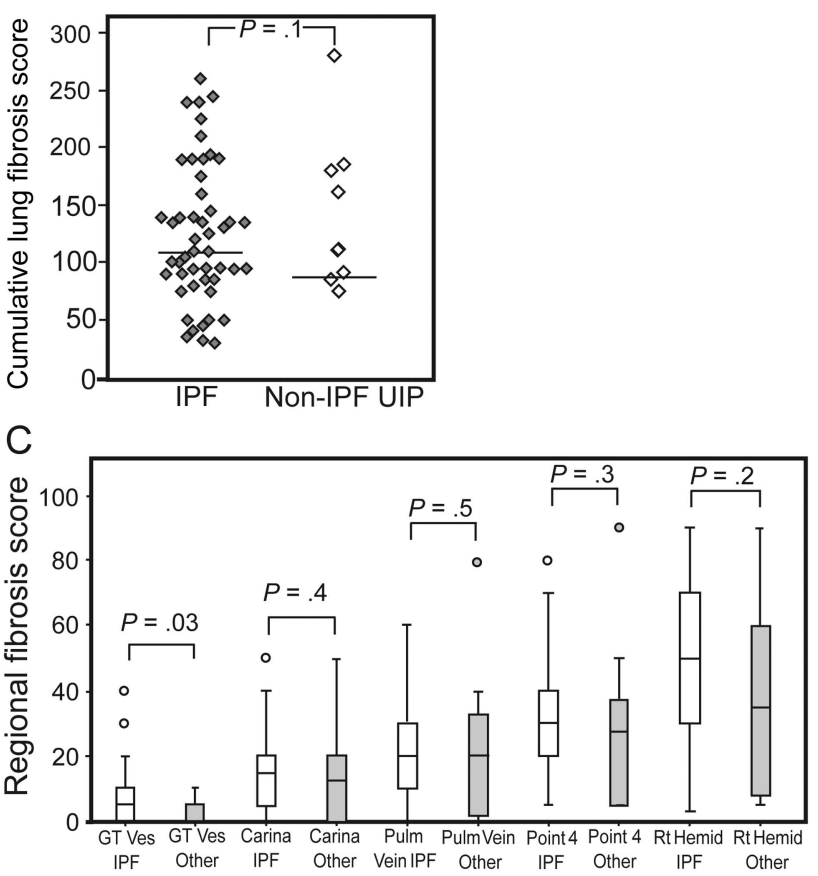

Fig. 2. Subgroups of subjects with emphysema and fibrosis (CPFE, $n=61$ ). A, mean pack-year, mean age, and number of male (M) versus female subjects are depicted on the abscissa for the CPFE population, parsed by IPF/non-IPF UIP status. $P$ value for gender analysis is two-tailed Fisher exact test; for the other analyses, $P$ values are two-tailed Student $t$ tests, with unequal variance. B, extent of fibrosis depicted by cumulative fibrosis scores for all 5 radiologic lung levels per subject. Each diamond represents a subject's cumulative score. Median values shown by horizontal lines in both subgroups. C, regional extent of fibrosis. For each radiologic lung level depicted on the ordinate, parsed by IPF/non-IPF UIP status, fibrosis scores are as shown. Box plot represents interquartile range and median, and whiskers represent data within 1.5 interquartile range of lower and upper quartiles. Outliers are shown as open circles. Two-tailed independent sample MannWhitney $U$ test $P$ values are shown, with $\alpha$ level post-Bonferroni correction at .01. Gt Ves $=$ at the level of the great vessels. Pulm $=$ pulmonary. Rt Hemid $=1 \mathrm{~cm}$ above right hemidiaphragm. IPF = idiopathic pulmonary fibrosis. UIP = usual interstitial pneumonia.

UIP/emphysema subjects exhibited a less steep gradient of transition from finer to coarser reticulation going from a cephalad to caudal direction, suggestive of biologic dif- ferences in regional susceptibility to reticulation in IPF/emphysema versus non-IPF/emphysema subjects (Fig. $4 C)$.

Regarding emphysema, the extent of emphysema was significantly greater in subjects with IPF/emphysema compared with non-IPF UIP/emphysema ( $P=.04$; Fig. 5A). At all 5 radiologic lung regions, concordant, statistically insignificant trends were observed toward higher regional emphysema extent in IPF emphysema subjects versus their non-IPF counterparts (Fig. 5B). Comparison of Figs. 5B and $4 \mathrm{~B}$ reveals that the lung level at which both emphysema burden and reticulation coarseness show the greatest significance is the level of the pulmonary veins, that is, the anatomic median of the 5 levels. At all 5 lung regions examined, there were at least some IPF/emphysema subjects with evidence of widespread involvement $(>25 \%$ emphysema involving the given radiologic level) that numbered fewer approaching the lung bases. For the 3 most caudal lung levels, there were no non-IPF UIP/emphysema subjects with evidence of such widespread emphysematous involvement $(P>.05$ for each of the 5 lung levels; Fig. 5 C). The proportion of subjects with $>25 \%$ emphysematous involvement of $\geq 1$ lung level was significantly greater in the IPF/emphysema subgroup (92\%) than in the non-IPF UIP/emphysema subgroup $(67 \%, P=.04$; Fig. 5D).

\section{Discussion}

Emphysema has a better understood relationship with smoking than does IPF, and recent clinical descriptions of the syndrome of CPFE have renewed interest in the interplay among these two smoking-associated disease processes. ${ }^{5,27-31}$ Currently, it is unclear whether there is any discrete pathobiological mechanism responsible for the natural history of CPFE, or are there merely co-existing fibrotic (eg, UIP) and emphysematous lesions present in those exposed to the shared environmental risk factor, smoke. ${ }^{32,33}$ A recent hypothesis suggests that fibrosis and emphysema share common pathogenetic mechanisms of accelerated senescence via telomere length abnormalities. ${ }^{34}$ Although data exist showing differences in fibrosis and emphysema involvement in IPF compared with other forms of UIP, the data are confounded by differing smoking status and other relevant confounders. ${ }^{16}$ The present study asked the question: is the burden of radiologic fibrosis and emphysema greater in smoke-exposed IPF subjects compared with similarly exposed non-IPF subjects with UIP? Patients with CT thorax imaging deemed not to be probable or definite UIP by defined international radiological criteria were excluded; this was important, given the prevalence of nonspecific interstitial pneumonia in patients with autoimmune interstitial lung disease. ${ }^{3,18,23,26}$ Using prospective radiologic rescoring of fibrotic and emphysema- 

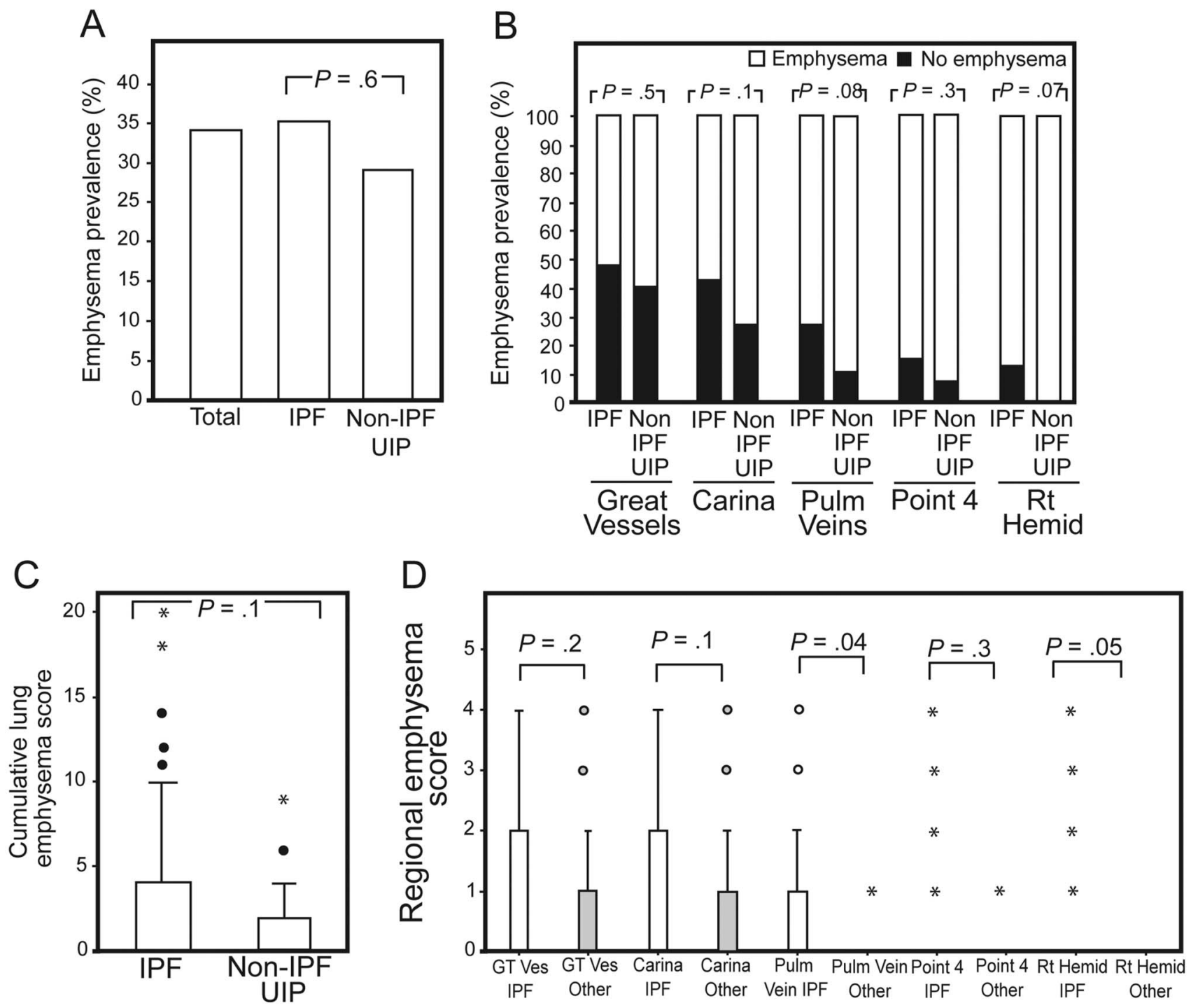

Fig. 3. Radiologic emphysema scores in IPF and non-IPF UIP groups. A, emphysema prevalence, as determined by having $\geq 5 \%$ emphysema in any of 5 radiologic lung levels per subject, is depicted on the ordinate as the percentage of such subjects among each indicated group. The vertical dashed line separates the total prevalence data on the left (in gray) from the comparative prevalence data for the two indicated groups on the right. B, similarly, emphysema prevalence data are presented for each of the 5 radiologic lung levels, parsed by IPF/non-IPF status, as the percentage of subjects that have emphysema. $P$ values represent unadjusted Mann-Whitney $U$ tests; $\alpha$ level for significance following Bonferroni correction is .01. C, extent of radiologic emphysema in the two study groups, shown as cumulative emphysema scores for all of the 5 radiologic lung levels per subject. Box plot represents interquartile range and median, and whiskers represent data within 1.5 interquartile range of lower and upper quartiles. Outliers shown as open circles and stars. D, regional extent of emphysema. For each radiologic lung level depicted on the ordinate, parsed by IPF/non-IPF UIP status, emphysema scores are as shown, using box plots as per panel C. Median emphysema score was zero for all groups assessed at the levels of point 4 and the right hemidiaphragm (see Methods). Mann-Whitney $U P$ values shown; $\alpha$ level post-Bonferroni correction for multiple testing is .01 . Gt Ves $=$ at the level of the great vessels. Pulm $=$ pulmonary. Rt Hemid $=1 \mathrm{~cm}$ above right hemidiaphragm. IPF $=$ idiopathic pulmonary fibrosis. UIP = usual interstitial pneumonia.

tous changes in current/former smokers with a prior UIP diagnosis, the data demonstrate greater fibrotic coarseness in IPF smokers than in smokers with secondary causes of UIP. The differences are not explained by studied confounding variables, either when the total IPF study population is compared with the non-IPF total population or when the corresponding subgroups with CPFE are com- pared with each other. The reticulation effects were most noticeably different when the IPF CPFE versus non-IPF CPFE subjects were compared, with the former subgroup having significantly greater cumulative reticulation scores. At all 5 regional lung levels, there were also concordant effects favoring coarser reticulation in IPF for a given amount of smoking compared with non-IPF, with the dif- 
A
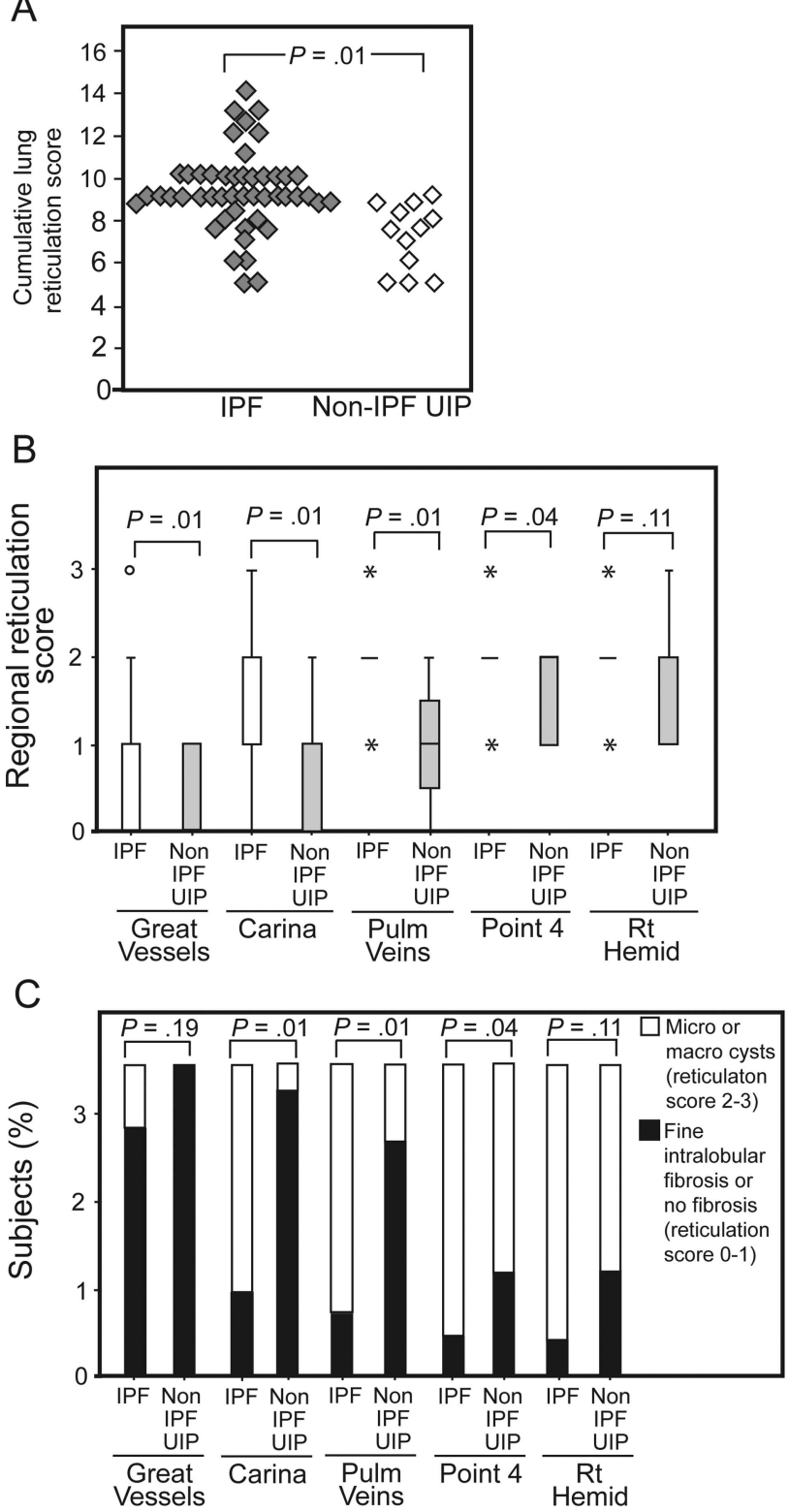

Fig. 4. Coarseness of fibrosis in CPFE subgroups. A, coarseness of fibrosis shown as cumulative reticulation scores for all 5 radiologic lung levels per subject, with adjustment of raw scores for uninvolved lung levels (see Methods). Each diamond represents a subject's cumulative score. B, regional coarseness. Reticulation scores are shown for each radiologic lung level indicated on the ordinate, parsed by IPF/non-IPF status. Box plot represents interquartile range and median, and whiskers represent data within 1.5 interquartile range of lower and upper quartiles. Outliers shown as open circles and stars. C, regional lung distribution of coarser fibrotic changes. Reticulation scores are assigned to two bins, coarser microcysts/macrocysts (reticulation scores of 2 or 3) and less coarse/no fibrosis (reticulation scores of 0 or 1 ), and are shown as the percentage of subjects within the CPFE subgroups of IPF and non-IPF UIP that have greater or lesser coarseness. All $P$ values are derived from Mann-Whitney $U$ tests. For panels $B$ and $C$, the $P$ values that are shown in bold font are significant following Bonferroni correction for multiple testing at $\alpha$ level of .01. ferences being stronger in (apical) lung regions that are typically involved at a more advanced stage of UIP. Furthermore, the data from the CPFE subgroups demonstrate a significantly greater extent of emphysema in IPF/emphysema than was observed in non-IPF UIP/emphysema, in the expected inverse lung regional distribution to that observed for the reticulation changes. The emphysema differences were not as strongly statistically significant as the reticulation changes, with weaker effects at a regional lung level. Together, these observations suggest a more radiologically extensive form of CPFE in those with IPF/emphysema (IPFE) compared with those with non-IPF UIP/emphysema, with implications for the pathobiology of IPF with emphysema and how future studies of CPFE should be designed.

It is widely accepted that an association exists among smoking and IPF with data exhibiting a healthy smoker effect and a negative influence of smoking on IPF outcome. ${ }^{30}$ What is less clear is whether smoking affects the UIP process similarly, irrespective of UIP etiology. In a study of IPF UIP and collagen vascular disease-associated UIP, the collagen vascular disease-associated UIP patients had less radiologic emphysema and a tendency for less honeycombing versus IPF. ${ }^{16}$ There were also significantly lower smoking rates and fewer men in the collagen vascular disease-associated UIP group versus IPF, confounding the overall observations. Data have now emerged showing a higher prevalence of concurrent radiologic emphysema in association with low pack-year smoking histories in patients with either IPF or rheumatoid arthritisassociated interstitial lung disease (RA-ILD) compared with COPD and non-COPD controls. In that study, there was no significant difference in the emphysema prevalence among the RA-ILD group compared with IPF, and the rheumatoid lung CPFE subgroup had higher coarseness scores than RA-ILD cases that lacked emphysema, suggesting the RA-ILD form of UIP is linked to smoking. It was also shown that the coarseness and extent of fibrosis was greater in IPF than in RA-ILD in that study, in the presence of similar smoking histories, which was also observed for IPF compared with non-IPF UIP in the current study. ${ }^{15}$ The present study also shows that, when other forms of non-IPF UIP are included in addition to RA-ILD, IPFE has greater fibrosis and emphysema scores than nonIPF CPFE, despite similar gender/age/smoking histories. This suggests that all forms of UIP are not the same, where interaction with smoking is concerned, with IPF being propagated more by smoking. Support for this contention is provided by the observation of others, that, when IPFE is excluded, radiologic coarseness scores are significantly higher in smokers with IPF versus nonsmokers with IPF. ${ }^{15}$ Although none of the pulmonary function parameters were significantly different among IPF versus non-IPF UIP groups, it is noteworthy that the strongest trend toward a 

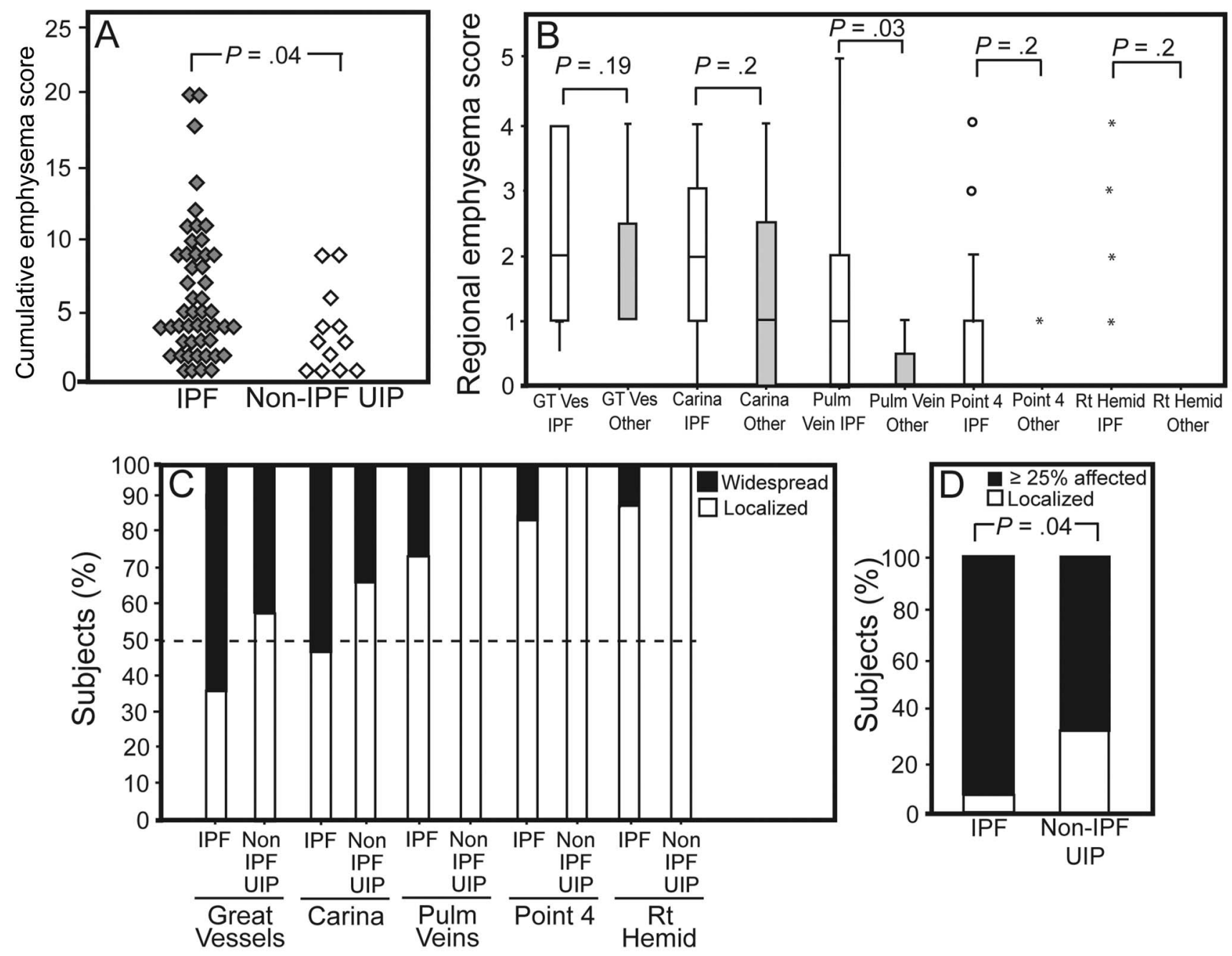

Fig. 5. Emphysema scores in CPFE subgroups. A, cumulative emphysema scores for the 5 radiologic lung levels are shown, comparing IPF emphysema to non-IPF UIP emphysema subjects. Mann Whitney $U P$ value is shown. B, emphysema scores are presented on the abscissa for each of the 5 indicated regional lung levels, comparing the IPF emphysema subjects to those emphysema subjects with non-IPF (Other) UIP. Mann-Whitney $U$ test $P$ values are shown, with post-Bonferroni $\alpha$ level of .01. C, regional lung distribution of more widespread emphysematous change. At each lung level indicated on the ordinate, the CPFE population (IPF emphysema vs non-IPF UIP emphysema) is presented, based on whether they have less extensive (and even absence of) emphysema at that given lung level (gray bars). D, the CPFE subpopulation $(n=61)$ is parsed by IPF/non-IPF status and by whether the subjects had only a lesser extent of emphysema, defined as only one radiologic level involved by emphysema that was present in no more than $25 \%$ of the level examined. $P$ value represents two-tailed Fisher exact test.

difference was observed for diffusing capacity. This could intuitively reflect the greater burden of fibrotic damage in the IPF group that was observed when assessed radiologically, and which could arguably have attained physiologic significance in a study of larger power.

Potential explanations for the current data are as follows: (1) an excessive traction or elastic force in IPF pulls open more and/or larger emphysematous holes compared with other forms of UIP; (2) subjects with IPFE have a pathobiologically distinct tendency toward greater reticulation and emphysematous destruction for the same smoke exposure compared with other causes of UIP with emphysema; (3) some of the true micro- or macrocystic reticulation changes are being miscategorized as emphysema or vice versa. We feel that the third interpretation is less likely, reflecting the radiology investigators' decision to avoid using automated software to quantify emphysema/honeycombing. Against the first and second interpretations are the observations that, in a lung regional level where regional cystic reticulation change was maximally coarser in IPFE versus non-IPF UIP/emphysema (at the carina), there was no significant difference in the corresponding emphysema scores. In favor of the first interpretation might be the observation that the lung regional 
level where the pairing of both reticulation and emphysema differences were most different (in IPF vs non-IPF UIP) was the level of the pulmonary veins, a somewhat intermediate level with respect to apex and base of lung. Arguably, this level might reflect an optimal equilibrium point, where there is sufficient axial cross-sectional area of both emphysema and reticulation involvement (emanating and converging from opposing poles of the lung) to best observe any difference in fibrotic traction effects on adjacent emphysema formation between the 2 study groups. Such a traction effect could be enhanced by a co-existing pathobiological predisposition toward emphysema formation in IPF, that is, both explanations 1 and 2. As some CPFE patients can have non-UIP fibrosis/emphysema, ${ }^{11}$ and to avoid semantic confusion, we propose the term idiopathic pulmonary fibrosis with emphysema (IPFE) be used to refer to the apparently synergistic combination of IPF and emphysema described herein.

The retrospective identification of subjects and smoking histories (possible selection- and lead-time biases), singlecenter involvement, and small size of the secondary UIP group (potential for inadequate statistical power) should lead to some caution in interpretation of results. All 132 subjects had a confident CT thorax diagnosis of UIP and had undergone multidisciplinary assessment. Of the 132 subjects included in the present study, a total of 18 (13.6\%) had undergone surgical lung biopsy, and all 18 revealed a pathologic finding of UIP. Although higher rates of surgical lung biopsies are described in clinical trials of IPF, at an international meeting of IPF experts, it was noted that, in routine practice, such biopsies are performed in $<15 \%$ of patients, ${ }^{35}$ which is possibly due to clinician pessimism that lung biopsy findings will alter the treatment plan. ${ }^{36}$ Nonetheless, study strengths include blinded prospective consensus scoring of all CT data in a high-throughput national referral center for ILD and connective tissue diseases, and time restriction of CT data to exclude older CT methodologies employed at our institution, which, in any event, appears to have minimal influence on emphysema and fibrotic scores. ${ }^{37}$ The proportion of patients with pulmonary fibrosis who also have emphysema ranges from $8 \%$ to $50.9 \%, 6,8,9,31,38$ depending on definitions/inclusion criteria, and the high prevalence of CPFE observed in the current study $(61 / 132=46 \%)$ reflects a $\geq 5$-pack-year history inclusion criterion and a more permissive radiologic emphysema definition ( $\geq 5 \%$ in any of 5 lung levels), which we felt was appropriate to our research question. It is possible that, if IPF smokers were separately compared with groups of etiologically pure secondary UIP smokers (eg, asbestosis alone or rheumatoid-associated ILD alone), there could be some discordant comparisons found to those presented herein; however, due to the low numbers of non-IPF UIP subjects in the present study, we planned to pool all secondary forms of UIP as was the case. The present study may have lacked power to address the issue of emphysema prevalence in IPF and secondary UIP. There are conflicting reports of the relative prevalence of emphysema in RA-ILD smokers versus IPF smokers, which may reflect selection/lead time biases or confounding by smoking burden, and only the present study compares emphysema burden within IPFE versus other CPFE subgroups. ${ }^{15,16}$

\section{Conclusions}

The present study shows that IPF with emphysema shows radiologically worse fibrosis and emphysema compared with non-IPF UIP with emphysema, which is not explained by key confounding variables, and suggests that the combination of IPF and emphysema is more than the sum of its parts. The current findings suggest that future studies of CPFE should stratify for IPFE separately, and account for smoking burden. The data lend support to the hypothesis that there is a synergy among IPF and emphysema in smokers, which may reflect inherent susceptibility to worse emphysema in IPFE and/or greater traction forces on emphysematous lesions in IPFE, and merits further study.

\section{REFERENCES}

1. Auerbach O, Garfinkel L, Hammond EC. Relation of smoking and age to findings in lung parenchyma: a microscopic study. Chest 1974;65(1):29-35.

2. Keller CA, Naunheim KS, Osterloh J, Espiritu J, McDonald JW, Ramos RR. Histopathologic diagnosis made in lung tissue resected from patients with severe emphysema undergoing lung volume reduction surgery. Chest 1997;111(4):941-947.

3. Flaherty KR, Thwaite EL, Kazerooni EA, Gross BH, Toews GB, Colby TV, et al. Radiological versus histological diagnosis in UIP and NSIP: survival implications. Thorax 2003;58(2):143-148.

4. Cottin V, Cordier JF. The syndrome of combined pulmonary fibrosis and emphysema. Chest 2009;136(1):1-2.

5. Cottin V, Nunes H, Brillet PY, Delaval P, Devouassoux G, TillieLeblond I, et al. Combined pulmonary fibrosis and emphysema: a distinct underrecognised entity. Eur Respir J 2005;26(4):586-593.

6. Jankowich MD, Rounds S. Combined pulmonary fibrosis and emphysema alters physiology but has similar mortality to pulmonary fibrosis without emphysema. Lung 2010;188(5):365-373.

7. Wang Q, Takashima S, Wang JC, Zheng LM, Sone S. Prevalence of emphysema in individuals who underwent screening CT for lung cancer in Nagano prefecture of Japan. Respiration 2001;68(4):352356.

8. Schmidt SL, Nambiar AM, Tayob N, Sundaram B, Han MK, Gross $\mathrm{BH}$, et al. Pulmonary function measures predict mortality differently in IPF versus combined pulmonary fibrosis and emphysema. Eur Respir J 2011;38(1):176-183.

9. Akira M, Yamamoto S, Inoue Y, Sakatani M. High-resolution CT of asbestosis and idiopathic pulmonary fibrosis. Am J Roentgenol 2003; 181(1):163-169.

10. Samara KD, Margaritopoulos G, Wells AU, Siafakas NM, Antoniou KM. Smoking and pulmonary fibrosis: novel insights. Pulm Med $2011 ; 461439$. 


\section{IPF WITH EMPHYSEMA}

11. Jankowich MD, Polsky M, Klein M, Rounds S. Heterogeneity in combined pulmonary fibrosis and emphysema. Respiration 2008; 75(4):411-417.

12. Cottin V. [Syndrome of combined pulmonary fibrosis and emphysema: understanding the functional profile.] Rev Mal Respir 2013; 30(3):173-175.

13. Marigliano B, Soriano A, Margiotta D, Vadacca M, Afeltra A. Lung involvement in connective tissue diseases: a comprehensive review and a focus on rheumatoid arthritis. Autoimmun Rev 2013;12(11): 1076-1084.

14. Gutsche M, Rosen GD, Swigris JJ. Connective tissue disease-associated interstitial lung disease: a review. Curr Respir Care Rep 2012; 1:224-232.

15. Antoniou KM, Walsh SL, Hansell DM, Rubens MR, Marten K, Tennant R, et al. Smoking-related emphysema is associated with idiopathic pulmonary fibrosis and rheumatoid lung. Respirology 2013; 18(8):1191-1196.

16. Song JW, Do KH, Kim MY, Jang SJ, Colby TV, Kim DS. Pathologic and radiologic differences between idiopathic and collagen vascular disease-related usual interstitial pneumonia. Chest 2009;136(1):2330.

17. Copley SJ, Wells AU, Sivakumaran P, Rubens MB, Lee YC, Desai SR, et al. Asbestosis and idiopathic pulmonary fibrosis: comparison of thin-section CT features. Radiology 2003;229(3):731-736.

18. Lynch DA, Godwin JD, Safrin S, Starko KM, Hormel P, Brown KK, et al. High-resolution computed tomography in idiopathic pulmonary fibrosis: diagnosis and prognosis. Am J Respir Crit Care Med 2005; 172(4):488-493

19. Johkoh T, Sakai F, Noma S, Akira M, Fujimoto K, Watadani T, Sugiyama Y. Honeycombing on CT; its definition, pathologic correlation, and future direction of its diagnosis. Eur J Radiol 2014; 83(1):27-31.

20. Ando K, Sekiya M, Tobino K, Takahashi K. Relationship between quantitative CT metrics and pulmonary function in combined pulmonary fibrosis and emphysema. Lung 2013 Dec;191(6):585-591.

21. Cottin V. Significance of connective tissue diseases features in pulmonary fibrosis. Eur Respir Rev 2013;22(129):273-280.

22. Goldblatt F, O'Neill SG. Clinical aspects of autoimmune rheumatic diseases. Lancet 2013;382(9894):797-808.

23. Desai SR, Veeraraghavan S, Hansell DM, Nikolakopolou A, Goh NS, Nicholson AG, et al. CT features of lung disease in patients with systemic sclerosis: comparison with idiopathic pulmonary fibrosis and nonspecific interstitial pneumonia. Radiology 2004;232(2):560567.

24. Hansell DM, Bankier AA, MacMahon H, McLoud TC, Müller NL, Remy J. Fleischner Society: glossary of terms for thoracic imaging. Radiology 2008;246(3):697-722
25. Wisser W, Klepetko W, Kontrus M, Bankier A, Senbaklavaci O, Kaider A, et al. Morphologic grading of the emphysematous lung and its relation to improvement after lung volume reduction surgery. Ann Thorac Surg 1998;65(3):793-799.

26. Litmanovich D, Boiselle PM, Bankier AA. CT of pulmonary emphysema: current status, challenges, and future directions. Eur Radiol 2009;19(3):537-551

27. Antoniou KM, Hansell DM, Rubens MB, Marten K, Desai SR, Siafakas NM, et al. Idiopathic pulmonary fibrosis: outcome in relation to smoking status. Am J Respir Crit Care Med 2008;177(2):190-194.

28. Wiggins J, Strickland B, Turner-Warwick M. Combined cryptogenic fibrosing alveolitis and emphysema: the value of high resolution computed tomography in assessment. Respir Med 1990;84(5):365369.

29. Doherty MJ, Pearson MG, O’Grady EA, Pellegrini V, Calverley PM. Cryptogenic fibrosing alveolitis with preserved lung volumes. Tho$\operatorname{rax}$ 1997;52(11):998-1002.

30. Ryerson CJ, Vittinghoff E, Ley B, Lee JS, Mooney JJ, Jones KD, et al. Predicting survival across chronic interstitial lung disease: the ILD-GAP model. Chest 2014;145(4):723-728.

31. Ryerson CJ, Hartman T, Elicker BM, Ley B, Lee JS, Abbritti M, et al. Clinical features and outcomes in combined pulmonary fibrosis and emphysema in idiopathic pulmonary fibrosis. Chest 2013;144(1): 234-240.

32. Cottin V. Interstitial lung disease. Eur Respir Rev 2013;22(127):26-32.

33. Munson JC. Combined pulmonary fibrosis and emphysema: a highpressure situation. Eur Respir J 2010;35(1):9-11.

34. Papiris SA, Triantafillidou C, Manali ED, Kolilekas L, Baou K, Kagouridis K, Bouros D. Combined pulmonary fibrosis and emphysema. Expert Rev Respir Med 2013;7(1):19-31; quiz 32.

35. Pulmonary Fibrosis Foundation. IPF Summit 2011: from bench to bedside. http://www.docstoc.com/docs/76760115/Overview-andRationale-Idiopathic-pulmonary-fibrosis_IPF_is-a. Accessed October 9, 2014.

36. King, TE Jr. Clinical advances in the diagnosis and therapy of the interstitial lung diseases. Am J Respir Crit Care Med 2005;172(3): 268-279.

37. Wells AU, Desai SR, Rubens MB, Goh NS, Cramer D, Nicholson AG, et al. Idiopathic pulmonary fibrosis: a composite physiologic index derived from disease extent observed by computed tomography. Am J Respir Crit Care Med 2003;167(7):962-969.

38. Kurashima K, Takayanagi N, Tsuchiya N, Kanauchi T, Ueda M, Hoshi $\mathrm{T}$, et al. The effect of emphysema on lung function and survival in patients with idiopathic pulmonary fibrosis. Respirology 2010;15(5):843-848 